\title{
Commentary on Regular Primary Care Lowers Hospitalisation Risk and Mortality in Seniors with Chronic Respiratory Disease
}

\author{
Barbara Starfield, MD, MPH \\ Department of Health Policy and Management, Johns Hopkins University, Baltimore, MD, USA.
}

J Gen Intern Med 25(8):758-9

DOI: $10.1007 /$ s11606-010-1393-y

(c) Society of General Internal Medicine 2010

$\mathrm{E}$ vidence of the benefits of primary care is robust ${ }^{1,2}$. Primary health care as a unique delivery system entity provides more effective, equitable, and efficient care and, as a clinical mode of health services delivery, its four functions of primary care (first contact, person-focused care over time ("longitudinality"), comprehensiveness of services, and coordination) have demonstrable beneficial effects on a variety of health-related phenomena including better recognition of patients' problems, more accurate diagnosis, better concordance with appointment keeping and with treatment advice, less emergency department use, fewer potentially avoidable hospitalizations, better generic (not specifically diseaseoriented) prevention, better monitoring of abnormalities, fewer medication prescriptions, and fewer unmet needs ${ }^{3}$. The benefits are much greater when the primary care provider is the same individual over time, as compared with a relationship with a particular place, except for preventive care (presumably because the need for routine preventive care is easily documented by transfer of information by means other than interpersonal contact, e.g., medical records) ${ }^{3}$.

What is it about "regularity" of care that might contribute to one or more of these functions of primary care? The study by Einarsdottir and colleagues ${ }^{4}$ was predicated on the assumption that regular contact with a general practitioner facilitates early symptom recognition and consequent adjustment of treatment as needed.

Does regularity of care facilitate early problem recognition? For it to work in this way, the "regularity" would have to involve consistency of care over time with a particular practitioner. As noted above, the benefits of longitudinality are much greater when it is provided by a particular practitioner rather than merely with a particular place. Although symptom recognition is rarely (if ever) a target of efforts to improve quality of care, it is a critical and rate-limiting step to the other processes of clinical intervention: adequate diagnosis, management, and reassessment. It takes knowledge of a patient (rather than just a disease or type of disease) to achieve better problem recognition ${ }^{5}$. When patients and their clinicians agree on what the patients' problems are, the patient is more likely to be improved on follow-up whether judged by the patient or the practitioner. Recognition of patients' problems from one time to

Published online May 19, 2010 another is best when the same practitioner is involved both times, less well when the same type of practitioner (e.g., physician-physician, nurse-nurse) is involved, even less well when a nurse follows up after a physician, and worst when a physician follows-up after a nurse) ${ }^{5}$. Therefore, there is some justification for the assumption that regular care would facilitate problem recognition if the provider is familiar with the patient as a result of a long-term relationship. In the study by Einarsdottir et al., there is no information on whether the care is usually or always provided by the same practitioner; the regularity observed in the data set only reflects the duration of time between visits. Wolinsky and colleagues ${ }^{6}$ recently showed that non-continuity of the primary care physician, as defined as more than eight months between visits to the same primary care physician, is associated with increased mortality over a fifteen year period in individuals 70 years of age or older. We cannot be sure that the benefit of "regularity" is a result of the establishment of better interpersonal relationship over time without knowing whether the practitioner remains the same. Would the same effect have been observed if the regular contact had been by phone, by mail (email or otherwise), or by routine follow-up with an unfamiliar auxiliary health worker who might change from time to time? Certainly, any of these mechanisms would have been less costly than a face-to-face visit with a physician. The findings of this study provide a powerful impetus to explore the mechanism(s) by which the benefit of regularity of visits occurs and the different ways of achieving it. Does it depend on the frequency of visits? On the continuity of the practitioner? Or just the effect of periodic contact? (And if so, what should the "period" be?

Some of the findings are puzzling. Why was there no doseresponse effect in which the more the regularity, the better the outcomes? For neither mortality nor hospitalization is there a clear gradient effect. Is the comorbidity measure an adequate one? Subsequent studies could use measures that outperform the Charlson index along clinically relevant dimensions ${ }^{7}$. Key outcomes (hospitalizations and premature death) are likely to be influenced substantially by the nature of the medication used. It appears that the main benefit of regular visits on risk of hospitalization is among patients prescribed low doses of inhaled corticosteroids, either with or without long-acting bronchodilators. The effects of regular care on all-cause mortality are unimpressive, as they fail to show a doseresponse effect (more regular care associated with lower death rates) when the patients were stratified by the type of medication. In fact, those on short-acting bronchodilators who have more regular care seem to have higher all-cause mortality. In seeking to learn about the effects of "regularity" on monitoring of medications over the long term, we should ask if regularity can avert adverse events in people with and without 
comorbidity and prevent medication-related adverse events among those who do and those who do not adhere to protocols.

An important contribution of this paper is the insight that chronic illnesses have acute manifestations requiring much more than routine monitoring. There is nothing about chronic illnesses that makes them unlike acute illnesses in their need for ongoing responsiveness to patients' needs. The now-vast literature on the management of chronic conditions neglects acute exacerbations. Virtually all of the studies of the impact of strategies such as the Chronic Care Model (CCM) leave the impression that the major challenge of management is monitoring to assure compliance with medication and selfcare management. This study explicitly raises the issue of early warning signs that have received little attention in the chronic care management literature.

Perhaps subsequent analyses of the data can help to understand the mechanism of the effect of regular care. Is the benefit equity-producing, that is, preferentially beneficial to people with more limited social means, or is it disparitycreating because of being more easily used by people with fewer social needs? Is the benefit equal for those whose regular visits are initiated by the health professionals and for those who are simply encouraged to seek early care when they develop symptoms? Is it possible to distinguish visits that are regularly scheduled by providers for "checkups" from those that are at the initiation of the patient? In which situation is the benefit greatest? The study provided no information on the mechanism for achieving regularity. Did it occur by providing set appointments for patients or was it achieved by encouraging patients to return as soon as possible when they think they are experiencing a problem? Is there an added benefit from pre-set appointments, as compared with making appointments more easily available? The nature of visits to primary care is changing, at least in the US; about half of all visits to general internists are now for routine follow-up ${ }^{8}$, but there is little evidence that routine care is uniformly beneficial. Greater benefit might be achievable by mechanisms that rely on better responsiveness of practitioners to patient-perceived problems - the essence of patient-centered care over time.

What about the influence of regularity of primary care on number and type of specialist visits? Are they increased, decreased, or unchanged when there is greater regularity of primary care visits? In view of the increasing use of specialists in the US health services system, this is an important issue. The more primary care physicians that are seen, even after controlling for number of visits, the greater the use of specialists and the greater the use of all types of resources and $\operatorname{costs}^{9}$. Does it matter if the effect of regularity is with a variety of primary care doctors and/or specialists? One (presumably unexpected) effect of a chronic care model demonstration was an increase in referrals to specialists ${ }^{10}$.
The authors of this study are to be commended for raising a new issue in health services research. They have scratched the surface of a potentially important mechanism for improving care. But the absence of a clear gradient in benefit from least regular to most regular raises questions about potential mediating variables. There is more work to be done to explain and explore the nature of the effects and their modifiers. Does regularity achieve benefits through better knowledge of the whole patient, better ability to detect deviations from everyday symptoms, more rapid response to symptoms, better knowledge and management of coexisting morbidity, better management of ancillary resources, better responsiveness of patients to recommended interventions, better ability of patients themselves to challenge care that does not seem right to them, better acceptance of needed changes in daily activities, better awareness among patients of worsening symptoms, all of the above, or something else? We need no further demonstrations of the benefits of person-centered care over time. What we need now is to know whether these achievements are better accomplished with more routine care or with better and more rapid responsiveness to patients' problems, and what types of professionals are best for these aspects of primary care.

Corresponding Author: Barbara Starfield, MD, MPH; Department of Health Policy and Management, Johns Hopkins University, 624 North Broadway, room 452, Baltimore, MD 21205, USA (e-mail: bstarfie@jhsph.edu).

\section{REFERENCES}

1. Starfield B, Shi L, Macinko J. Contribution of primary care to health systems and health. Milbank Q. 2005;83:457-502.

2. Kringos DS, Boerma WG, Hutchinson A, van der Zee J, Groenewegen PP. The breadth of primary care: a systematic literature review of its core dimensions. BMC Health Serv Res. 2010;10:65.

3. Starfield B. Primary Care: Balancing Health Needs, Services and Technology. New York: Oxford University Press; 1998.

4. Einarsdottir K, Preen D, Emery J, Kelman C, Holman C. J General Intern Med. doi:10.1007/s11606-010-1361-6.

5. Starfield B. Primary care and equity in health: the importance to effectiveness and equity of responsiveness to peoples' needs. Hum Soc. 2009;33:56-73

6. Wolinsky FD, Bentler SE, Liu L, et al. Continuity of care with a primary care physician and mortality in older adults. J Gerontol A Biol Sci Med Sci. 2010;65:421-8.

7. The Johns Hopkins University ACG Case-Mix System (http://www.acg. jhsph.edu, accessed April 14, 2010).

8. Valderas JM. Analyses of 2009 National Ambulatory Medical Care Survey data (personal communication). 2010

9. Starfield B, Chang H, Lemke KW, Weiner JP. Ambulatory specialist use by non-hospitalized patients in US health plans: correlates and consequences. J Ambul Care Manage. 2009;32:216-25.

10. Reid RJ, Fishman PA, Yu O, et al. Patient-centered medical home demonstration: a prospective, quasi-experimental, before and after evaluation. Am J Manag Care. 2009;15:e71-87. 